\title{
Personality Profiles of Individuals with Substance Use Disorders: Historical Overview and Current Directions
}

\author{
Jeffrey S. Nevid*, Alexander J. Gordon, Andrew S. Miele, Luke H. Keating
}

St. John's University, New York

Article Info

Article Notes

Received:April 7, 2020

Accepted: May 27, 2020

\section{*Correspondence:}

Jeffrey S. Nevid, Ph.D, Department of Psychology, St. John's

University, Queens, NY 11439; Email: nevidj@stjohns.edu.

(c) 2020 Nevid JS. This article is distributed under the terms of the Creative Commons Attribution 4.0 International License.

\section{Abstract}

Efforts to understand personality features of people who use psychoactive substances have a long history, dating back to early psychoanalytic conceptualizations. Advancements in the field have focused on applying multidimensional personality inventories to better understand personality differences between substance users and non-users, and between different substance use types, with respect to both psychopathological traits and broad dimensional factors. A brief review of this evidence highlights personality features of persons with alcohol and other substance use problems and between users of different types of substances, especially alcohol and opioid substance use disorder patients. A better understanding of personality profiles of substance use disorder groups may be useful in tailoring treatment approaches based on profile characteristics.

Medical and psychological research literature documents a long history of attempts to identify and characterize personality profiles associated with problematic substance use. Approaches to this question have varied, utilizing objective, projective, dimensional, and categorical measures of personality as well as psychoanalytic formulation of substance use behavior. Studies initially focused on examining evidence for the existence of an "alcoholic" or "addictive" personality structure that may predispose individuals to engage in problematic alcohol and drug use.

Research efforts have shifted away from attempts to identify an overriding concept of a single personality type ${ }^{1}$. More recent work seeks to develop etiologic predictive models that identify and compare personality profiles of users and nonusers and between users of different substances. Furthermore, these newer approaches have increasingly used more complex and integrative models incorporating both biological and socioeconomic factors. The current paper provides a brief narrative review of research evidence exploring personality profiles of individuals with substance use disorders as well as differences in personality traits among users of different substances. Understanding differences in personality patterns may help inform targeted intervention efforts to address personality-level factors that underpin substance use and risk factors in substance use disorders, which may differ among individuals who use different substances.

\section{Psychoanalytic Approaches}

Early psychoanalytic approaches emphasized drives toward libidinal gratification and defenses against aggressive impulses as the basis for addiction and substance use $\mathrm{e}^{2-4}$. Moreover, fixation at 
the oral stage of psychosexual development was thought to lead to development of oral-dependent personality traits, such as dependence and depression, as well as to behavioral patterns in adulthood aimed at oral gratification, such as excessive drinking or smoking.

Although persons who develop alcohol use disorder or other forms of substance misuse may show dependent traits, it remains unclear whether dependence leads to alcoholism or stems from downward movement in social status associated with problem drinking, largely as a result of unemployment, which likely renders people more dependent on others. Nor does demonstrating a statistical association between dependence and alcoholism establish that alcoholism represents an oral fixation or can be traced to problems in early psychosexual development. It is noteworthy that later psychodynamic models of personality functioning of substance users put a greater emphasis on deficits in ego strength and self-regulation driving efforts toward self-medication of emotional distress ${ }^{5}$. More recently, there has been a shift in focus from understanding personality dynamics in substance use to using objective personality inventories to identify personality profiles associated with substance use and substance use disorders. Moreover, contemporary researchers have focused more attention on comorbidity between personality disorders and substance use disorders.

\section{Personality Profiles}

Researchers have consistently demonstrated links between substance use and diagnoses of borderline and antisocial personality disorders ${ }^{6-9}$. Although antisocial personality disorder is often associated with patterns of drug use and misuse, debate has centered around its lack of specificity in predicting substance use behavior due to the high rates of antisocial personality features among non-users ${ }^{10}$. In part due to the predictive limitations of diagnosis-level information, a growing body of literature has favored trait-level dimensional conceptions of personality in developing personality profiles of individuals with substance use disorders.

The MacAndrew Alcoholism Scale (MAC) of the Minnesota Multiphasic Personality Inventory (MMPI) is an early notable example of a dimensional approach to measurement of personality traits used to predict problematic substance use potential ${ }^{11}$. The scale was developed using 44 items from the original version of the MMPI that, although not related to drinking behaviors per se, were differentially endorsed by alcohol users as compared to normative samples. Although originally developed to predict proneness to alcoholism, studies have found the MAC to be nonspecifically associated with use of other substances, such as opioids, as well as with markers of addictive behaviors related to problem eating behaviors (i.e., obesity, anorexia) ${ }^{12,13}$. Factor analysis of the MAC delineated six subscales: Risk Taking, Cognitive Impairment, School Maladjustment, Interpersonal Competence, Extroversion and Exhibitionism, and Moral Indignation $^{11}$. High scores on the MAC are related to impulsivity, issues with authority and antisocial tendencies, and extraversion, representing a substrate of personality traits often associated with problem drinking and other problematic substance use $\mathrm{e}^{14}$.

Cloninger's $(1987)^{15}$ tridimensional model of personality has been particularly influential in the development of etiological models identifying personality patterns in substance use populations. Each dimension of the model (novelty-seeking, harm-avoidance, and rewarddependence) has been demonstrated to be predictive of later substance use based on childhood measures ${ }^{16}$. Cloninger's typology of alcoholism subtypes provides a useful heuristic framework for distinguishing different alcoholic types based on substance use behaviors, underlying biological processes, and distinguishing personality features. The type I alcoholic personality, which is associated with use of alcohol as self-medication and an anxious or neurotic personality profile, is frequently characterized by low novelty seeking (NS), high harm avoidance (HA) and high reward dependence $(\mathrm{RD})^{17}$. The type II alcoholic personality, described in terms of antisocial personality features and characterized by impulsivity, is often associated with the opposite personality profile (high NS, low HA, and low RD).

Other researchers also emphasize heterogeneity within populations of individuals with substance use disorders. For example, Babor and colleagues ${ }^{18}$ conducted a cluster analysis with a sample of individuals with alcohol use disorder. Their findings showed two definable clusters having distinct risk factors, personality profiles, and course of disease. Other researchers have explored dimensional factors relating to substance use, converging on a profile characterized by high levels of impulsivity and disinhibition and negative emotionality ${ }^{19-22,25,58}$. These constructs have been linked to several aspects of substance misuse, including severity of dependence, age of onset, and substance use etiology ${ }^{21}$. Applying a five-factor model, levels of alcohol involvement are frequently associated with low levels of conscientiousness, agreeableness, and high levels of neuroticism ${ }^{22,23}$. These traits are in line with earlier research on etiological models of alcohol use ${ }^{16}$. Researchers have also adopted an integrative, multidimensional view of personality traits associated with substance use $\mathrm{e}^{24,25}$. For example, investigators incorporated biological correlates and gene-environment interactions that contextualize personality and substance use behavior ${ }^{26,27}$.

Multidimensional personality inventories have been introduced that better capture a wider range of personality traits in relation to substance use disorders, including 
inventories such as Multidimensional Personality Questionnaire (MPQ) and the Personality Assessment Inventory (PAI). For example, our research groups used multidimensional personality inventories to examine psychopathological personality traits in substance use disorder groups ${ }^{28}$.

Research in personality psychopathology has also identified clusters of traits associated with antisocial and borderline personality disorders as risk factors for future alcohol use ${ }^{29}$. Across studies and types of substances, researchers find that problematic substance use is highly comorbid diagnostically with personality disorders in general, and with antisocial and borderline personality traits in particular ${ }^{30}$.

Lower-level trait analyses allow for a richer and more complex personality architecture to emerge ${ }^{31}$. For example, disinhibited behavior can be represented in terms of two distinct, yet related, components: impulsivity and sensation-seeking ${ }^{32}$. Each of these trait components has been related to substance use etiology and outcomes and are often treated as separate, subordinate traits in research studies $^{33}$. More research is needed using hierarchical models that incorporate multimodal assessment of both clinical and nonclinical personality traits ${ }^{34}$.

Studies focusing on lower-order traits find personalityspecific relations between facets of neuroticism, specifically hopelessness and anxiety-sensitivity, and disinhibition, which is typically described in terms of impulsivity and sensation-seeking ${ }^{32}$. A study of personality traits relating to motives to drink found that factors such as anxietysensitivity and feelings of hopelessness were uniquely correlated with motives to use substances to self-medicate and reduce negative affect ${ }^{24}$.

A study applying the five-factor model (FFM) identified similarities in personality traits across subgroups of users of cocaine and heroin ${ }^{35}$. MANCOVA analysis was conducted with user-status as an independent variable, demographic factors as covariates, and personality traits as dependent variables. The results indicated that across user subgroups, users tended to score high on measures of neuroticism and low on conscientiousness. In a meta-analysis of current marijuana users, pooled effect size estimates showed users scoring low on agreeableness and conscientiousness, yet while also reporting average levels of neuroticism and high levels of openness to experience ${ }^{36}$.

More recently, investigators examined prescription drug use among young adults between the ages of 18 and 26, using latent profile analysis to identify different risk strata within this population ${ }^{37}$. Comparisons of mean differences between latent groups on responses to addiction scales identified distinct patterns of personality traits. While anxiety-sensitivity was associated with use of sedatives and sensation-seeking was linked to stimulant use, impulsivity was related to misuse across all types of prescription medications.

\section{Biobehavioral and Developmental Models}

Explanations for relations between disinhibition and substance use have also begun to incorporate integrative, developmental models. Cloninger's model was the first to propose that genetic predisposition may link substance use and personality traits, a claim which subsequent research has supported ${ }^{27}$. Significant overlap has been found between genetic variance and personality traits linked to substance use, such as behavioral control and agreeableness $^{26,34}$.

Extensive research on risk factors for substance use beyond personality profiles has been conducted across different groups of users, both in specific samples (e.g., college-age drinkers) and representative samples (e.g., national comorbidity surveys). Overall, support for demographic risk factors has been equivocal ${ }^{38}$. For example, studies have shown that males tend to have a higher prevalence of drinking behavior than females ${ }^{39}$. Meanwhile, the observed racial differences in drinking behavior are often attributed to socioeconomic factors, such as income and education, and availability of substances rather than differences in race alone ${ }^{40,41}$.

Equally important as individual risk factors, then, may be the developmental context in which personality operates and substance use patterns form. The impact of this pathway can be studied in terms of environmental self-selection, or the ability of individuals to shape their environments in ways that match their personality ${ }^{42}$. A longitudinal study of self-selection into Greek life and problem drinking among college students found both direct and indirect effects of personality traits on drinking behaviors. Using a latent growth model, the researchers found direct effects of personality and drinking behaviors were demonstrated by individuals with higher levels of extraversion tending to self-select into Greek life-a college lifestyle associated with high-risk drinking-even after controlling for levels of pre-college drinking. Alternatively, higher neuroticism predicted pre-college drinking and subsequent entrance into fraternities or sororities ${ }^{42}$. These results point to the interplay between personality and environmental influences in the development and maintenance of problem drinking.

\section{Comparisons of Personality Traits of Substance Users and Non-Users}

Researchers report distinct profiles of personality traits in comparing substance users and non-users ${ }^{43}$. With respect to general dimensions of personality, substance users tend to display elevated levels of reactivity, negative 
affect, and novelty-seeking. In terms of personality pathology, drug users tend to display elevations in antagonism, disinhibition, and psychoticism as compared to non-users. Furthermore, these personality trait patterns are often associated with diagnoses of borderline and antisocial personality disorders, classifications frequently comorbid with substance use disorders ${ }^{43}$.

Personality profiles of users of substances other than alcohol are characterized primarily by low levels of conscientiousness, with equivocal support for the role of other traits ${ }^{44}$. Relations between personality traits and nicotine use reveal a similar profile to that of alcohol use, with low conscientiousness, low agreeableness, and high neuroticism emerging as the strongest predictors of smoking behavior ${ }^{23}$.

\section{Personality Trait Comparisons of Users of Different Types of Substances}

While research evidence on differences in personality traits among users of different types of substances is diverse with respect to the personality constructs measured and substance types compared, the literature can be distinguished in terms of analyses of clinical or pathological personality traits (e.g., MMPI, PAI, diagnostic scales) and more general or normative personality dimensions, such as those represented by "Big Five" model traits. The most common comparisons are those involving individuals with alcohol and opioid (typically heroin) use disorders.

\section{Comparisons Based on Psychopathological Personality Traits}

An early MMPI study used discriminant function analysis to compare personality functioning of alcohol and heroin users admitted to an inpatient unit, finding that alcohol users exhibited a profile more consistent with neurotic features than did opioid users ${ }^{45}$. This profile comprised syndromes such as anxiety, depression, and somatization. Opioid users, however, demonstrated higher levels of defensiveness, activity, and ego strength, presenting with more self-confidence and mood stability, but with the presence of features related to social deviance (e.g., egocentricity, nonconformity, and impulsivity). While a degree of social deviance was apparent for both groups, these traits were predominate among opioid users.

Evidence also indicates that among users admitted for inpatient treatment of substance use problems, those who misuse opioids are more likely to exhibit externalizing symptoms and personality disorders than those who misuse alcohol or marijuana. For example, Hopwood, Baker, and Morey (2008) ${ }^{46}$ used exploratory factor analysis, finding that among inpatients in a substance use treatment program, heroin and cocaine users exhibited higher levels of externalizing symptoms on the PAI (predominantly antisocial behavior) than did control, alcohol, or marijuana groups. In contrast to the earlier work of Sutker and colleagues ${ }^{45}$, Hopwood's group found that heroin and cocaine groups also scored higher on an internalizing factor (predominantly depression and anxiety) than did controls or alcohol or marijuana groups. Using discriminant function analysis, our research group found similar results with the PAI, showing that opioid use disorder patients had higher levels than alcohol use disorder patients on both internalizing (i.e., anxiety, anxiety-related disorders) and externalizing (antisocial and borderline features) pathology ${ }^{47}$. We also found that opioid patients scored higher on suicidal ideation than alcohol patients, although their scores on average were not clinically elevated. The net result of these recent studies is to raise awareness of more extensive internalizing and externalizing pathological traits in opioid use disorder patients.

Supporting the more profound personality pathology in opioid patients, researchers have also noted that opioid and other drug users are more likely to meet criteria for clinical diagnosis of borderline, antisocial, dependent, and narcissistic personality disorders as compared to alcohol users $^{29,48,49}$. Despite this trend, some researchers find little evidence to distinguish alcohol and opioid users in terms of early maladaptive schemas originating from adverse childhood experiences ${ }^{50}$.

Lending more support to these differences, an early MMPI study used discriminant function analysis to compare personality traits in different types of substance users, showing that alcohol users tended to be least disturbed when compared to cocaine, heroin, and polysubstance users, with the latter groups exhibiting the most severe dysfunctional personality traits, including paranoia, withdrawal, and anxiety ${ }^{51}$. These investigators also distinguished heroin users as more depressed and alienated in contrast to cocaine users who showed disturbed personality traits primarily characterized by impulsivity.

\section{Comparisons Based on General Personality Dimensions}

Research applying Cloninger's model and using logistic regression analysis showed that while both alcohol and opioid dependent patients in residential treatment tended to score high on Novelty-Seeking, opioid users were significantly more extreme on this trait ${ }^{48}$. When compared to those with alcoholism, opioid users also exhibited higher levels of self-transcendence but lower levels of harm avoidance. Two profiles emerge from this work, one comprising easily bored, exploratory, stimulus-seeking opioid users and a second comprising more emotionally vulnerable alcohol users seeking relief from negative emotions. 
Several research efforts focusing on impulsivity using the Barratt Impulsiveness Scale Version $11^{52}$ found no meaningful differences between alcohol and opioid users, although both groups had higher levels of impulsivity than controls ${ }^{53,54}$. Using logistic regression, one of these studies suggests that different domains of impulsivity may better characterize different substance use types, as alcohol users were distinguished from controls on motor impulsivity, whereas opioid users were distinguished on non-planning impulsivity ${ }^{53}$. This, too, is consistent with distinctions between stimulus-seeking (opioid) versus vulnerable and anxious (alcohol) users.

Discount rates, which represent the degree of reduced value due to a delay in receiving a reward, have also been used in the literature as a proxy for impulsiveness. Research along these lines shows that although heroin and cocaine users do not tend to differ from each other in discount rates, they both tend to exhibit higher discount rates (lower reward value for a longer delay) than alcohol users ${ }^{55}$.

Research applying Big Five personality traits shows that alcohol users tend to be lower on extraversion than other substance use groups, indicating a more reserved personality and less social connectiveness ${ }^{54}$. Other investigators find that alcohol and polydrug users do not differ significantly on levels of neuroticism and openness to experience; however, polydrug users tend to score lower than non-users on conscientiousness and agreeableness while alcohol users do not ${ }^{56}$. These investigators also found that polydrug user profiles were more consistent with antisocial personality disorder than were those of alcohol users, with higher levels of Sensation-Seeking, Disinhibition, Experience Seeking, and Thrill and Adventure Seeking.

Other researchers applied the three higher-order personality dimensions of the Multidimensional Personality Questionnaire (MPQ) as a proxy for the Big Five traits ${ }^{57}$. This research showed that substance users tend to differ the most on constraint, which corresponds to Big Five factors of (high) conscientiousness and (low) openness to experience. Alcohol users showed higher levels of constraint than did opioid users, and alcohol and marijuana users combined showed higher levels of constraint than opioid and cocaine users combined. Constraint also shared a negative relationship with a diagnosis of antisocial personality disorder, a finding consistent with other research that personality disorders and psychopathy are more prevalent among opioid users.

One of the limitations in examining personality differences among substance use types is the confound of age. Alcohol users in addictions research tend to be older than those who use other drugs, prompting some researchers to control for age while others accept the age differential on grounds of ecological validity because it represents typical patterns in the general population ${ }^{57,45-47}$.
The current review utilizes a narrative approach to examining historical, methodological, and theory-based development and current trends related to investigating personality profiles of individuals with substance use disorders and personality differences based on substance of choice. We recognize that a limitation of this approach is the difficulty of synthesizing a large body of research that reflects different perspectives from different time periods in the field's history as well as the diversity in demographic characteristics of the populations studied.

In summary, evidence presented in this review bears on differences in personality traits between substance users and nonusers and among different types of substance use types. An important clinical implication of research on differences between substance use types is the understanding that a "one-size-fits-all" approach should not be used as a guide to treatment. Evidence of personality differences relating to different substance use types, especially the more severe levels of pathological personality traits generally found among opioid users, supports the need to take personality differences into account as a guide to individualize treatment in the interest of targeting clinical improvement.

\section{References}

1. Wood MD, Vinson DC, Sher KJ. Alcohol use and misuse. In A. Baum, T. A. Revenson, \& J. E. Singer (Eds.), Handbook of health psychology Mahwah, NJ: Lawrence Erlbaum Associates. 2001; 280-320.

2. Abraham K. The psychological relations between sexuality and alcoholism. International Journal of Psycho-Analysis. 1926; 7: 2-10.

3. Freud S. A case of hysteria, three essays on sexuality and other works (1905). In J. Strachey (Ed.), The standard edition of the complete psychological works of Sigmund Freud, Volume VII (1901-1905). London: Hogarth Press and the Institute of Psychoanalysis. 1953.

4. Glover E. On the aetiology of drug-addiction. International Journal of Psycho-Analysis. 1932; 13: 298-328.

5. Khantzian EJ. Understanding addictive vulnerability: An evolving psychodynamic perspective. Neuropsychoanalysis. 2003; 5(1): 5-21.

6. Grande TP, Wolf AW, Schubert DS, et al. Associations among alcoholism, drug abuse, and antisocial personality: A review of literature. Psychological Reports. 1984; 55(2): 455-474.

7. Hasin D, Kilcoyne B. Comorbidity of psychiatric and substance use disorders in the United States: Current issues and findings from the NESARC. Current Opinion in Psychiatry. 2012; 25(3): 165-171. doi:10.1097/YCO.0b013e3283523dcc

8. Langås AM, Malt UF, Opjordsmoen S. In-depth study of personality disorders in first-admission patients with substance use disorders. BMC Psychiatry. 2012; 12(1): 180. https://doi.org/10.1186/1471244X-12-180

9. Sullivan T, Frances J. Substance use in borderline personality disorder. American Journal of Psychiatry. 1990; 147: 1002-1007.

10. Nathan PE. The addictive personality is the behavior of the addict. Journal of Consulting and Clinical Psychology. 1988; 56(2): 183-188. https://doi.org/10.1037/0022-006X.56.2.183

11. Schwartz MF, Graham JR. Construct validity of the MacAndrew Alcoholism Scale. Journal of Consulting and Clinical Psychology. 1979; 47(6): 1090-1095. https://doi.org/10.1037/0022-006X.47.6.1090 
12. Lachar D, Berman W, Grisell JL, et al. The MacAndrew Alcoholism Scale as a general measure of substance misuse. Journal of Studies on Alcohol. 1976; 37(11): 1609-1615.

13. Leon GR, Kolotkin R, Korgeski G. MacAndrew Addiction Scale and other MMPI characteristics associated with obesity, anorexia and smoking behavior. Addictive Behaviors. 1979; 4(4): 401-407.

14. Allen JP. Personality correlates of the MacAndrew Alcoholism Scale: A review of the literature. Psychology of Addictive Behaviors. 1991; 5(2): 59-65. https://doi.org/10.1037/h0080609

15. Cloninger CR. A systematic method for clinical description and classification of personality variants: A proposal. Archives of General Psychiatry. 1987; 44(6): 573-588. doi:10.1001/ archpsyc.1987.01800180093014

16. Cloninger CR, Sigvardsson S, Bohman M. Childhood personality predicts alcohol abuse in young adults. Alcoholism: Clinical and Experimental Research. 1988; 12(4): 494-505.

17. Cloninger CR, Sigvardsson S, Bohman M. Type I and type II alcoholism: An update. Alcohol Health \& Research World. 1996; 20(1): 18-23.

18. Babor TF, Grant M. (Eds). Project on identification and management of alcohol-related problems: Report on phase II: A randomized clinical trial of brief interventions in primary health care. Geneva: World Health Organization, Programme on Substance Abuse. 1992.

19. Alcorn JL, Gowin JL, Green CE, et al. Aggression, impulsivity, and psychopathic traits in combined antisocial personality disorder and substance use disorder. The Journal of Neuropsychiatry and Clinical Neurosciences. 2013; 25(3): 229-232. https://doi.org/10.1176/appi. neuropsych. 12030060

20. Baldacchino A, Balfour DJK, Matthews K. Impulsivity and opioid drugs: Differential effects of heroin, methadone and prescribed analgesic medication. Psychological Medicine. 2015; 45(6): 11671179. https://doi.org/10.1017/S0033291714002189

21. Ball SA, Cobb-Richardson P, Connolly AJ, et al. Substance abuse and personality disorders in homeless drop-in center clients: symptom severity and psychotherapy retention in a randomized clinical trial. Comprehensive Psychiatry. 2005; 46(5): 371-379.

22. Lejuez CW, Magidson JF, Mitchell SH, et al. Behavioral and biological indicators of impulsivity in the development of alcohol use, problems, and disorders. Alcoholism Clinical and Experimental Research. 2010; 34: 1334-1345.

23. Malouff J, Thorsteinsson E, Schutte N. Smoking and the five-factor model of personality: A meta-analysis. Journal of Drug Education. 2006; 36: 47-58.

24. Comeau N, Stewart SH, Loba P. The relations of trait anxiety, anxiety sensitivity, and sensation seeking to adolescents' motivations for alcohol, cigarette, and marijuana use. Addictive Behaviors. 2001; 26(6): 803-825.

25. Whiteside SP, Lynam DR. The five factor model and impulsivity: Using a structural model of personality to understand impulsivity. Personality and Individual Differences. 2001; 30(4): 669-689.

26. De Moor MH, Vink JM, van Beek JH, et al. Heritability of problem drinking and the genetic overlap with personality in a general population sample. Frontiers in Genetics. 2011; 2: 76.

27. Slutske WS, Heath AC, Madden PA, et al. Personality and the genetic risk for alcohol dependence. Journal of Abnormal Psychology. 2002; 111(1): 124

28. Nevid JS, Gordon AJ, Haggerty G. Clinical utility of the Personality Assessment Inventory in predicting symptom change and clinical outcome in an inpatient chemical dependency rehabilitation unit. Journal of Personality Assessment. 2019; 1-7. https://doi.org/10.10 80/00223891.2019.1627665

29. Grant, B. F., Chou, S. P., Goldstein, R. B., et al. Prevalence, correlates, disability, and comorbidity of DSM-IV borderline personality disorder: Results from the Wave 2 National Epidemiologic Survey on Alcohol and Related Conditions. The Journal of Clinical Psychiatry. 2008; 69(4): 533-545. https://doi.org/10.4088/jcp.v69n0404

30. Elkins IJ,KingSM, McGue M, etal.Personality traitsand the development of nicotine, alcohol, and illicit drug disorders: Prospective links from adolescence to young adulthood. Journal of Abnormal Psychology. 2006; 115(1): 26-39. https://doi.org/10.1037/0021-843x.115.1.26

31. Watson D, Clark LA, Harkness AR. Structures of personality and their relevance to psychopathology. Journal of Abnormal Psychology. 1994; 103(1): 18-31.

32. Woicik PA, Stewart SH, Pihl RO, et al. The substance use risk profile scale: A scale measuring traits linked to reinforcement-specific substance use profiles. Addictive Behaviors. 2009; 34(12): 10421055.

33. Conrod PJ, Castellanos-Ryan N, Mackie C. Long-term effects of a personality-targeted intervention to reduce alcohol use in adolescents. Journal of Consulting and Clinical Psychology. 2011; 79(3): 296.

34. Littlefield AK, Stevens AK, Sher KJ. Impulsivity and alcohol involvement: multiple, distinct constructs and processes. Current Addiction Reports. 2014; 1(1): 33-40.

35. Terracciano A, Löckenhoff CE, Crum RM, et al. Five-Factor Model personality profiles of drug users. BMC Psychiatry. 2008; 8(1): 22. https://doi.org/10.1186/1471-244X-8-22

36. Gorman DM, Derzon JH. Behavioral traits and marijuana use and abuse: A meta-analysis of longitudinal studies. Addictive Behaviors. 2002; 27: 193-206.

37. Marmet S, Studer J, Rougemont-Bücking A, et al. Latent profiles of family background, personality and mental health factors and their association with behavioural addictions and substance use disorders in young Swiss men. European Psychiatry. 2018; 52: 76-84.

38. Inchley J, Currie D. Growing up unequal: Fender and socioeconomic differences in young people's health and well-being. Health Behaviour in School-aged Children (HBSC) Study (2013/2014 survey). World Health Organization International. 2013. https://doi.org/http:// www.euro.who.int/en/publications/abstracts/growing-up-unequal.hbsc-2016-study-20132014-survey

39. Chen P, Jacobson KC. Developmental trajectories of substance use from early adolescence to young adulthood: Gender and racial/ethnic differences. Journal of adolescent health. 2012; 50(2): 154-163.

40. Lillie-Blanton M, Anthony JC, Schuster CR. Probing the meaning of racial/ethnic group comparisons in crack cocaine smoking. JAMA. 2011; 269(8): 993-997.

41. Galea S., Nandi A, Vlahov D. The social epidemiology of substance use. Epidemiologic Reviews. 2004; 26(1): 36-52. https://doi. org/10.1093/epirev/mxh007

42. Park A, Sher KJ, Wood PK, et al. Dual mechanisms underlying accentuation of risky drinking via fraternity/sorority affiliation: The role of personality, peer norms, and alcohol availability. Journal of Abnormal Psychology. 2009; 118(2): 241.

43. Ruiz MA, Pincus AL, Schinka JA. Externalizing pathology and the fivefactor model: A meta-analysis of personality traits associated with antisocial personality disorder, substance use disorder, and their cooccurrence. Journal of Personality Disorders. 2008; 22(4): 365-388.

44. Bogg T, Roberts BW. Conscientiousness and health-related behaviors: A meta-analysis of the leading behavioral contributors to mortality. Psychological Bulletin. 2004; 130(6): 887-919. https://doi. org/10.1037/0033-2909.130.6.887

45. Sutker PB, Archer RP, Brantley PJ, et al. Alcoholics and opiate addicts. Comparison of personality characteristics. Journal of Studies on Alcohol. 1979; 40(7): 635-644. https://doi.org/10.15288/ jsa.1979.40.635 
46. Hopwood CJ, Baker KL, Morey LC. Personality and drugs of choice. Personality and Individual Differences. 2008; 44(6): 1413-1421. https://doi.org/10.1016/j.paid.2007.12.009

47. Nevid JS, Gordon AJ, Barris A, et al. Personality profiles of patients with alcohol use disorder and opioid use disorder in an inpatient treatment setting. Journal of Substance Abuse Treatment. 2019; 97: 91-96. https://doi.org/10.1016/j.jsat.2018.11.013

48. Milivojevic D, Milovanovic SD, Jovanovic M, et al. Temperament and character modify risk of drug addiction and influence choice of drugs: Personality factors in drug addiction. The American Journal on Addictions. 2012; 21(5): 462-467. https://doi.org/10.1111/j.15210391.2012.00251.x

49. Skodol AE, Oldham JM, Gallaher PE. Axis II comorbidity of substance use disorders among patients referred for treatment of personality disorders. American Journal of Psychiatry. 1999; 156(5): 733-738.

50. Shorey RC, Stuart GL, Anderson S. Differences in early maladaptive schemas in a sample of alcohol-and opioid-dependent women: Do schemas vary across disorders? Addiction Research \& Theory. 2013; 21(2): 132-140. https://doi.org/10.3109/16066359.2012.703266

51. Donovan JM, Soldz S, Kelley HF, et al. Four addictions: The MMPI and discriminant function analysis. Journal of Addictive Diseases. 1998; 17(2): 41-55. https://doi.org/10.1300/J069v17n02_04

52. Patton JH, Stanford MS, Barratt ES. Factor structure of the Barratt impulsiveness scale. Journal of Clinical Psychology. 1995; 51(6): 768-774.
53. Bozkurt M, Evren C, Yilmaz A, et al. Aggression and impulsivity in different groups of alcohol and heroin dependent inpatient men. Klinik Psikofarmakoloji Bülteni-Bulletin of Clinical Psychopharmacology. 2013; 23(4): 335-344. https://doi. org/10.5455/bcp.20130127021314

54. Zilberman N, Yadid G, Efrati Y, et al. Personality profiles of substance and behavioral addictions. Addictive Behaviors. 2018; 82: 174-181. https://doi.org/10.1016/j.addbeh.2018.03.007

55. Kirby KN, Petry NM. Heroin and cocaine abusers have higher discount rates for delayed rewards than alcoholics or non-drug-using controls. Addiction. 2004; 99(4): 461-471. https://doi.org/10.1111/j.13600443.2003.00669.x

56. Lackner N, Unterrainer HF, Neubauer AC. Differences in Big Five personality traits between alcohol and polydrug abusers: Implications for treatment in the therapeutic community. International Journal of Mental Health and Addiction. 2013; 11(6): 682-692.

57. Conway KP, Swendsen JD, Rounsaville BJ, et al. Personality, drug of choice, and comorbid psychopathology among substance abusers. Drug and Alcohol Dependence. 2002; 65(3): 225-234. https://doi. org/10.1016/S0376-8716(01)00168-5

58. Sher KJ, Trull TJ, Bartholow B, et al. Personality and alcoholism: Issues, methods, and etiological processes. In H. Blane \& K. Leonard (Eds.), Psychological theories of drinking and alcoholism 2nd ed. New York: Plenum. 1999; 55-105. 